\title{
Salivary dysfunction caused by medication usage
}

\author{
O.M. EINHORN ${ }^{1}$, K. GEORGIOU ${ }^{2}$ and A. TOMPA ${ }^{3 *}$
}

${ }^{1}$ Private Dental Office: Dr. Einhorn, 89 Medinat Hayehudim Street, Herzliya, Israel

${ }^{2}$ Private Dental Office: Dr. Georgiou, 2-4 Makarios Avenue, Nicosia, 1065, Cyprus

${ }^{3}$ Institute of Public Health, Semmelweis University, Budapest, 1089, Hungary

Received: March 17, 2020 • Accepted: May 11, 2020

Published online: July 24, 2020

(c) 2020 The Author(s)

\begin{abstract}
A considerable number of patients arriving in dental offices are being treated with ongoing medication for a variety of chronic diseases. As a result, dentists must be familiar with the potential side effects these therapeutic agents may have on the tissues of the oral cavity, and in particular on the salivary gland. Salivary gland function may be altered by a wide range of medications, leading to effects such as xerostomia, hyposalivation, hypersalivation or even swelling of the glands. These disorders can cause a variety of other health complications. This review will focus on the most common groups of drugs responsible for salivary gland dysfunction, including psychoactive drugs, antidepressants, antipsychotics, antihypertensives, and antihistamines.
\end{abstract}

\section{KEYWORDS}

salivary glands dysfunction, medication side effects, xerostomia, hyposalivation, hypersalivation

\section{INTRODUCTION}

Patients under chronic medication therapy are common, and dentists must be aware of the possible side effects caused by different drugs, as well as the mechanisms by which these medications act. A large number of both prescribed and non-prescribed drugs taken for the treatment of chronic diseases of the cardiovascular, urogenital, respiratory, neurologic, gastrointestinal systems (among others) may affect oral health. For example, many of the medications

* Corresponding author. Semmelweis University, Institute of Public Health, Orczy út 2-4, Budapest, 1089, Hungary. Tel.: +36 1210 2954x56196, E-mail: tompa.anna@med.semmelweis-univ.hu 


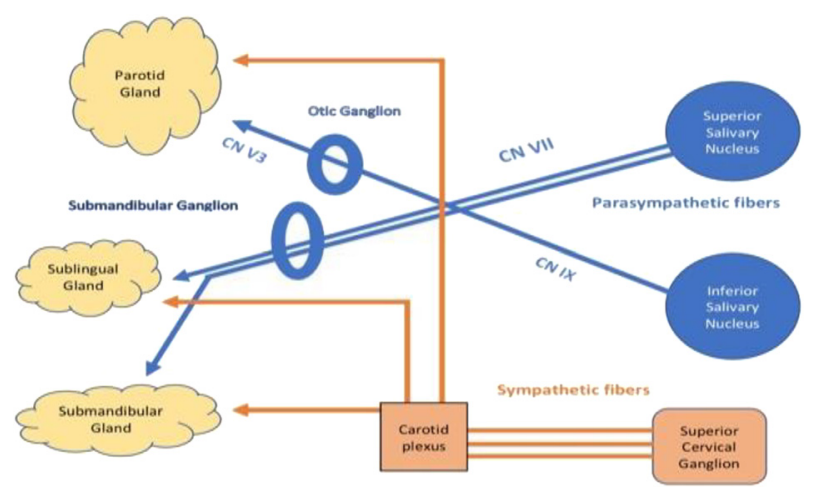

Fig. 1. Innervation of salivary glands. Shown are the sympathetic and parasympathetic pathways innervating the different salivary glands. Sympathetic innervation of all salivary glands arises from the superior cervical ganglion after which fibers pass through the carotid plexus to their target organs. Parasympathetic innervation is more complex. Nerve fibers in the parotid gland originate from the inferior salivary nucleus, and are carried by the glossopharyngeal nerve (CN IX). These fibers pass through the otic ganglion, and are then carried by auriculotemporal nerve of the mandibular division of the trigeminal nerve (CN V3). Parasympathetic nerve fibers in the submandibular and sublingual glands originate in the superior salivary nucleus and are carried by the chorda tympani nerve of Facial nerve (CN VII)

can cause xerostomia, caries and other lesions to the oral mucosa [1]. The most frequently reported complaint related to medication usage is salivation dysfunction, and specifically dry mouth sensation.

Salivation is defined as the secretion of fluids by the salivary glands, and is essential in several physiological processes, including digestion, food lubrication, buffering capacity, wound healing, defense against chemical and mechanical injuries, and the maintenance of the oral cavity [1]. The major salivary glands in the human body are the parotid glands, the submandibular glands and the sublingual glands. Saliva is an exocrine secretion, consisting of electrolytes and proteins including enzymes, antimicrobial proteins, glycoproteins, immunoglobulins, growth factors, and mucins, however the precise composition of saliva varies based on which gland it originates from. The salivary glands are largely regulated by the autonomic nervous system (ANS); parasympathetic and sympathetic nerves control the secretion of saliva by acting through receptors found on salivary acinar cells (Fig. 1) [2]. In addition to the control of the glands by the ANS, some gastrointestinal hormones can also affect the composition and flow rate of saliva [3].

Secretion of the two major components of saliva is a result of distinct stimulation by the ANS. Parasympathetic stimulation of the acinar cells results in secretion of the fluid component, including ions, while sympathetic stimulation of the glands leads to secretion of the protein component [1]. Secretion of chloride and sodium contributes to the production of an isotonic fluid by the acinar cells. There are five subtypes of muscarinic acetylcholine receptors, and the one that is responsible for the parasympathetic neurotransmission of the salivary glands is the M3 muscarinic receptor. Acetylcholine binds the M3 receptor located on the acinar cells, stimulating secretion of water and sodium chloride forming an isotonic saliva. However, as saliva travels through the salivary ducts, sodium and chloride ions are reabsorbed and 
bicarbonate and potassium ions are secreted into the saliva from the ductal cells. These two processes turn the saliva into a hypotonic fluid as it enters the oral cavity [2].

The process of secretion is thought to be dependent on two mechanisms which act through either a calcium mediated pathway or a cyclic adenosine monophosphate (cAMP) mediated pathway [4]. The M3 receptor is a G-protein coupled receptor. After acetylcholine binds the receptor, the associated G-protein activates phospholipase C (PLC). PLC then hydrolyzes phosphatidylinositol 4,5-bisphosphonate (PIP2) into inositol 1,4,5-triphosphate (IP3) and diacylglycerol (DAG). As IP3 binds its receptor (IP3R), located on the endoplasmic reticulum (ER), calcium ions are released from the ER into the cytosol leading to the secretion of enzymes, fluid and ions.

In contrast, sympathetic stimulation of the acinar cells involves the adrenergic receptors. Receptors such as $\alpha 1$ and $\beta 1$ adrenergic receptors, $\mathrm{H} 2$ histamine receptors, etc. mediate their function via the cAMP-protein kinase A (PKA) pathway. Sympathetic stimulation of the acinar cells involves binding of either epinephrine or norepinephrine to an adrenergic receptor on the cell membrane. Subsequent activation of the G-protein leads to activation of adenylate cyclase and conversion of ATP to cAMP. The resulting increased intracellular concentration of cAMP leads to activation of PKA. PKA plays a major role in several cellular functions, including secretion of the protein component of saliva.

Other receptors in addition to the M3 receptor, such as the NK1 receptor also regulate salivation via the IP3/DAG pathway. In addition, the main inhibitory neurotransmitter in the central nervous system (CNS), GABA, is involved in decreasing fluid and amylase secretion by acting on the GABA-A receptor located on the salivary gland $[4,5]$.

The function and product of the salivary gland may be altered by a wide range of medications, leading to effects such as xerostomia, hyposalivation, hypersalivation or swelling of the glands. These undesirable effects can be related to lower concentrations of calcium and phosphate in the saliva as well as its decreased flow rate. Another contributing factor is decreased secretion of growth factors into saliva. Growth factors and salivary mucins secreted by the salivary glands play a major role in the preservation of the oral mucosa integrity and wound healing. One important characteristic of these growth factors is to trap water, which aids in preventing tissue dehydration and assisting regeneration. For example, epidermal growth factor is found in a variety of tissues throughout the human body, but is also essential in the salivary glands, where it has an important role in oral wound healing [6].

\section{HYPERSALIVATION}

Hypersalivation is a state of over-secretion of saliva by the salivary glands. It can eventually lead to sialorrhea which can cause social embarrassment and may also lead to other consequences, including choking and aspiration pneumonia. The main medications which have hypersalivation as a common side-effect are a subset of psychoactive drugs which are used both by prescription and illegally (Table 1). In addition to their effects on salivary flow rates, these drugs may also change the composition of the saliva. Hypersalivation is an end-result of several different effects of these drugs: depletion of noradrenaline from central stores, excessive stimulation of M3 and dopamine D2 receptors, as well as inhibition of $\alpha 2$ adrenergic receptors [7]. For example, clozapine is an atypical antipsychotic drug, prescribed for patients with treatment- 
Table 1. Medications causing hypersalivation

\begin{tabular}{lc}
\hline Family & Drug \\
\hline Antipsychotics & Clozapine \\
& Olanzapine \\
& Quetiapine \\
\hline
\end{tabular}

resistant schizophrenia and associated with an elevated risk of blood disorders and hypersalivation.

\section{HYPOSALIVATION AND XEROSTOMIA}

Xerostomia is defined as a subjective complaint of dry mouth. Xerostomia affects over $30 \%$ of patients older than 65 [8]. Logic might suggest that patients reporting xerostomia would show decreased salivary flow, but in many cases, patients do not have objective signs of hyposalivation. Xerostomia is an end-result of various causes and can harm the oral cavity. Hyposalivation is a non-subjective state and in most cases, it is accompanied by xerostomia. The average salivary flow rate under stimulated conditions is $1.5-2.0 \mathrm{~mL} / \mathrm{min}$ and is $0.3-0.4 \mathrm{~mL} / \mathrm{min}$ under unstimulated conditions. Hyposalivation is defined as stimulated and unstimulated salivary flow rates below 0.5 and $0.1 \mathrm{~mL} / \mathrm{min}$, respectively [9]. In addition to causing discomfort to the patient, hyposalivation likely affects the oral microbiome [10] and increases the probability of dental caries, fungal and bacterial infections, and oral lesions [6,7]. Patients with hyposalivation experience taste disturbance and exhibit difficulty masticating and swallowing.

Hundreds, if not thousands of drugs, can induce xerostomia as a side-effect. Some of these agents cause a subjective complaint of dry mouth, while others actually induce hyposalivation by multiple mechanisms, such as inhibition of M3 muscarinic receptors and $\alpha 1$ adrenergic receptors, inhibition of noradrenaline uptake, or stimulation of $\alpha 2$ adrenergic receptors [4, 7]. Many mechanisms of action have been suggested to contribute to xerostomia. Some drugs compete with acetylcholine in both sympathetic and parasympathetic ganglia while other drugs compete with the release of acetylcholine at the parasympathetic effector junction. Another mechanism occurs at the higher centers of the brain, where stimulation of a subset of adrenoreceptors in the frontal cortex can result in inhibitory effects on salivary nuclei. Certain medications can also produce xerostomia without having an effect on neural pathways (Table 2). For example, these medications are mostly inhaled and can produce sensation of mouth dryness without altering the salivary flow. Another hypothesis suggests that dry mouth sensation may result from decreased fluid volume and loss of electrolytes secondary to increased urination and dehydration caused by medication usage [9].

Among all the mechanisms described above, the anticholinergic mechanism is a primary contributor in many cases of hyposalivation. Among the most common drugs that can cause dry mouth by interfering with the ANS signaling involved in regulation of saliva production are several groups of antidepressants, antipsychotics, $\beta$ blockers and antihistamines. These drugs are widely used, and dry mouth is a real and common occurrence in patients treated for psychiatric, hypertensive and urinary problems [4, 7]. Further exploration of these drug groups is considered below. 
Table 2. Medications causing xerostomia

\begin{tabular}{ll}
\hline Family & Drug \\
\hline Antidepressants & MAOs, TCAs, SSRIs \\
Antihypertensives & Clonidine, Guanfacine, Reserpine, Methyldopa, Timolol, Atenolol, \\
& Metoprolol, Diltiazem, Verapamil, Nifedipine, Thiazides, \\
& Furosemide \\
Antipsychotics & Olanzapine, Clozapine, Haloperidol, Quetiapine, Risperidone, \\
& Donepezil \\
Bronchodilators & Salbutamole, Terbutaline, Sameterol, Formoterol, Indacaterol, \\
& Tiotropium, Ipratropium \\
Antimuscarinic agents for & Oxybutynin and Tolterodine \\
overactive bladder syndrome & Tizanidine, Baclofen \\
Centrally acting skeletal muscle & \\
relaxants & Cetirizine, Acrivastine, Astemizole, Loratadine, Mizolastine \\
Hntihistamines & Ranitidine, Omeprazole \\
proton-pump inhibitors & \\
Opioids and benzodiazepines & Morphine, Dihydrocodeine, Tramadol, Diazepam \\
Cytotoxic drugs & 5-flurourcail \\
\hline
\end{tabular}

\section{Antidepressants}

Antidepressants such as monoamine oxidase inhibitors (MAOIs) and tricyclic antidepressants (TCAs) can lead to increased serotonergic or noradrenergic effects. TCAs can also cause the inhibition of cholinergic, histaminic and $\alpha 1$ adrenergic receptors, resulting in xerostomia. A study investigating the effects of several antidepressants has found that TCAs can lead to a significant decrease in salivary flow rate. Additionally, these drugs can cause a decrease in the salivary concentration of sodium, and an increase in the concentration of potassium. These effects are a direct consequence of muscarinic receptor inhibition. In contrast, drugs in the SSRI (selective serotonin reuptake inhibitors) class had no effect on these parameters. Another study reviewing the effects of the TCA dothiepin in non-depressed patients confirmed that this drug can decrease the stimulated salivary flow rate of the parotid gland [11]. An additional consideration with TCAs is sex-related differences in responses. It has been suggested that women might have a lower rate of serotonin synthesis in the brain, and as a result, they could be more sensitive than men to the depressant effects of tryptophan depletion [12].

\section{Antihypertensives}

Clonidine, guanfacine, reserpine and methyldopa are examples of classic, centrally acting antihypertensives. These agents stimulate $\alpha 2$ adrenergic receptors in the brain stem, leading to peripheral sympathetic inhibition thus to decreased blood pressure. Currently, centrally acting antihypertensive drugs are not commonly used due to prominent side effects, including dry mouth, sedation, dizziness and edema. However, other anti-hypertensives can also cause xerostomia. For example, ganglion blockers and particularly $\beta$ adrenergic receptor antagonists (the so-called " $\beta$ blockers") such as timolol, atenolol and metoprolol may cause a dry mouth 
sensation. This effect is thought to result from the stimulation of $\alpha 2$ adrenergic receptors found on the salivary glands and in the CNS [4]. A study comparing the effects of non-selective and $\beta 1$-selective adrenergic receptor antagonists with placebo, showed that it was largely the composition of saliva that was affected by the $\beta$ adrenergic receptor antagonists rather than salivary flow rate. The most notable changes with $\beta$ adrenergic receptor antagonists were drastic decreases in total protein composition and amylase activity, while salivary flow rates remained within normal values.

Another principal group of hypertensive drugs is the angiotensin-converting enzyme (ACE) inhibitors. Drugs of this group have long been used as one of the most important pharmacological tools for the treatment of arterial hypertension, and are also used for other cardiovascular indications. In addition to its ability to convert angiotensin I, ACE also has kinase activity, which prevents the accumulation of tissue mediators such as bradykinin. When this enzyme is inhibited, bradykinin molecules accumulate, leading to a number of adverse reactions. According to the available data, approximately $8-13 \%$ of patients using ACE inhibitors report xerostomia [13].

Diuretics are the most commonly prescribed drugs for cardiovascular disease and hypertension in the USA, and act by affecting the reabsorption of sodium in the kidneys, thus influencing the blood volume and pressure. The thiazide subgroup of diuretics act via the inhibition of the sodium-chloride cotransporter, found in the distal convoluted tubules of the nephron. The loop diuretics also act on sodium regulation in the kidneys are the loop diuretics. Furosemide, a loop diuretic agent, targets the sodium-potassium-chloride cotransporter found on the thick ascending limb of the nephron [14]. In a study following the side effects of therapeutic agents in elderly patients, diuretics and psychotropics were the most common medications inducing xerostomia and caused similar reduction of mean salivary flow rates [15]. Another study evaluating the side effects of furosemide also reported oral dryness as a side effect. During the study, urine and salivary secretions were obtained from five normal males after the oral intake of either $0.5 \mathrm{mg} / \mathrm{kg}$ body weight of furosemide or placebo. As expected, patients administered the drug showed a dramatic increase in urinary output and increased reports of xerostomia. However, there were no significant differences in salivary flow rate, total salivary output, total protein amount in the saliva or ion concentrations in saliva in patients receiving the drug. These findings have led to the conclusion that furosemide had a more notable effect on the kidneys than on the salivary glands, and that the sensation of oral dryness in this case may not be related to the quantity of salivary output [16].

Other antihypertensive drugs with salivary side effects include the widely used calcium channel blockers such as diltiazem, verapamil and nifedipine. These agents can cause xerostomia due to their effects on calcium regulation which has an essential role in saliva secretion [17].

\section{Antipsychotics}

Antipsychotic drugs are widely known to cause extrapyramidal symptoms, the most common side effects for these drugs. These symptoms include continuous muscle contractions, motor restlessness, muscle rigidity, slower movements, tremors, and the appearance of irregular movements. The mechanism of action for these symptoms is the inhibition of dopamine D2 receptors, or the eradication of the neurotransmitter from the basal ganglia. The functional absence of dopamine often leads to a pathological state of the extrapyramidal system [18]. In 
addition to these extrapyramidal symptoms, some antipsychotic medications also possess anticholinergic properties. Although newer drugs have been developed, both old and new antipsychotic medications can lead to a wide range of side effects. Despite the fact some of the antipsychotic agents have a selective affinity for D2 receptors, they continue to exhibit anticholinergic effects. These anticholinergic effects have particular importance, as one of the main side effects reported by patients using these medications is xerostomia [19]. It has been demonstrated that a long term and continuous treatment of schizophrenia with conventional antipsychotics is highly linked to anticholinergic side effects including dry mouth. Olanzapine, an atypical antipsychotic, was found to have a positive therapeutic effect on schizophrenia patients, but adverse reactions such as dizziness and dry mouth were very prominent $[4,20]$. Despite the associated effects, this drug is highly recommended in the treatment of patients with schizophrenia and related psychoses. Another atypical antipsychotic agent, clozapine, shares similar biochemical and pharmacological characteristics with those of olanzapine. A study comparing the usage of clozapine and olanzapine, both atypical antipsychotic drugs, showed that patients treated with olanzapine had significantly fewer instances of adverse effects (4\%) than those who were treated with clozapine (14\%). Strikingly, one of the most reported side effects was increased salivation, which was observed more frequently in patients treated with clozapine [21]. A study comparing olanzapine with haloperidol, a typical antipsychotic drug, has shown that olanzapine causes xerostomia more frequently [22]. Similarly, another study of atypical antipsychotics such as quetiapine and risperidone reported dry mouth in $14.5 \%$ and $6.9 \%$ of cases, respectively, and dry mouth more prevalent in the quetiapine group than the placebo group [23].

Donepezil is an acetylcholinesterase inhibitor that is marketed for the treatment of Alzheimer's disease. This disease results in memory loss and behavioral deterioration and is associated with by a deficit of acetylcholine. However, this drug is also used as an antipsychotic, usually in combination with other antipsychotic agents. Donepezil can improve memory loss and has the ability to counteract the xerostomia caused by other antipsychotics. Unfortunately, donepezil can also trigger mania, a major risk that must be considered when weighing its benefits [24].

\section{Bronchodilators}

Bronchodilators are the most prominent class of medications used to manage chronic obstructive pulmonary disease (COPD), and are also critical for the management of asthma. These drugs cause relaxation of airway smooth muscle cells of the airways, leading to bronchodilation. One main subgroup of bronchodilators is the $\beta 2$ adrenergic receptor agonists, which are further grouped based on their time of onset and duration of action. Example drugs in in this class include the short acting albutamol and terbutaline, the longer acting salmeterol and formoterol, and the ultra-long acting indacaterol. Even though xerostomia is not a commonly reported side-effect, several agents in this group have a strong link to dental caries and periodontal diseases [25]. Another subcategory of airway-dilating agents are the anticholinergics. The two most well-known anticholinergics used as bronchodilators are tiotropium and ipratropium. They show high receptor selectivity for the M1 and M3 receptors and, in common with $\beta$-agonists, their main route of administration is inhalation [26]. According to a study exploring the adverse effect of anti-asthma drugs, dry mouth is a common side effect, and the most 
commonly reported side-effect of tiotropium was xerostomia (9.3\%). This is thought to be related to inhibition of muscarinic receptors on the salivary glands [27]. The link between anticholinergics and dry mouth were also confirmed in other studies assessing the side effects of anti-asthma medications [28]. In addition to the studies demonstrating correlation between bronchodilators and xerostomia, another study was conducted to examine the correlation between chronic medication use and 5-year dental caries incidence among the elderly. The hypothesis behind this study was that medications causing xerostomia could increase caries incidence via two mechanisms. The first suggested mechanism is that medications associated with xerostomia could increase dental caries by leading to a chronic decrease in salivary flow rate and decreased capacity of saliva to buffer acids in the oral cavity. The second hypothesis is that the occurrence of xerostomia would lead to consumption of sweet drinks and food in an effort to relieve the dry mouth sensation, and thus increase caries incidence. The results indicated that patients who were under anti-asthma drug treatment during the previous 5 years had presented more cases of coronal caries than control patients, and it has been concluded that this group of drugs is strongly associated with caries manifestation and with the appearance of xerostomia [29].

\section{Antimuscarinic agents for overactive bladder syndrome}

The physiological function of the urinary system is largely under the control of M2 and M3 muscarinic receptors found on the smooth muscle of the urinary bladder, called the detrusor muscle [4]. When stimulated, M2 receptors block adenyl cyclase, resulting in the inhibition of the sympathetically-induced relaxation, leading to smooth muscle contraction, and in several pathological conditions, M2 receptors can directly contribute to smooth muscle contraction. From a therapeutic point of view, simultaneous inhibition of M2 and M3 receptors would be optimal in ensuring the complete inhibition of smooth muscle contractions. Oxybutynin, a widely used M3 receptor antagonist, and other similar drugs, are the most widely used therapy for urinary system problems. Anticholinergic drugs used by people with overactive bladder syndrome have proven to show large improvement in symptoms [30], with oxybutynin and tolterodine the most widely used to treat this syndrome. However, the M3 receptors on the urinary bladder are not the only target for this group of medications, and those therapeutic agents can lead to many adverse effects including dry mouth due to inhibition of the M3 receptors located on the salivary glands [4]. A comparison of Tolterodine IR (immediate release) and Oxybutynin IR showed that the two agents have similar efficacy, but that Tolterodine IR was been better tolerated, in particular with regard to the incidence and severity of dry mouth [31]. Adverse reactions including dry mouth, constipation and even blurred vision, are closely related to the anticholinergic activity of these drugs and occur frequently in up to $25 \%$ of patients, depending on the dosage [32]. In recent years, research efforts have been focusing on the development of drugs with selectivity to the muscarinic receptors found in the bladder, causing fewer adverse effects such as dry mouth.

\section{Centrally acting skeletal muscle relaxants}

Tizanidine, a central $\alpha 2$ adrenergic receptor agonist, is widely used in the treatment of patients suffering from spasticity associated with multiple sclerosis or any damage to the CNS. Tizanidine depresses muscle reflexes via both spinal and supra-spinal pathways, but the precise 
mechanism of action of this drug is not fully understood. In a study assessing the side effects caused of tizanidine, the most common complaint was dry mouth [33]. In a study conducted to compare the effects of baclofen, another widely used muscle relaxant, with those of tizanidine, forty patients suffering from multiple sclerosis were randomized into baclofen or tizanidine groups and treated for six weeks. Critically, dry mouth was frequently reported in patients taking either medication [34]. In addition to its use in overactive bladder syndrome discussed above, oxybutynin also has spasmolytic properties so may be present in this patient population as well.

\section{Antihistamines}

Selective $\mathrm{H} 1$ antihistamines are the "gold standard" in treatment against symptoms arising from allergic rhinitis [35]. These drugs function as inverse agonists, rather than antagonists of histamine $\mathrm{H} 1$ receptors, members of the G-protein coupled receptor family. H1 antihistamines were originally developed more than 70 years ago from other anticholinergic drugs, but the older generation of $\mathrm{H} 1$ antihistamines penetrates the blood-brain-barrier and can cause a sedating effect and tiredness, together with impaired memory and lower ability to concentrate [36]. The drugs which belong to this group are small lipophilic molecules, and are associated with several side effects largely due to their ability to cross the blood-brain barrier and alter the physiologic cholinergic activity [35]. Medications belonging to the second generation of selective $\mathrm{H} 1$ antihistamines are safer, reportedly causing fewer side effects [36]. This generation of antihistamines possesses more lipophobic properties, preventing the molecule from penetrating the blood brain barrier and thereby blocking CNS and cholinergic effects. In addition, this generation of medications has a longer duration of action allowing a more patient-friendly dosing schedule, which may result in increased patient compliance with therapy [35]. In a study investigating sedating vs. non-sedating antihistamines, the older antihistamines caused pronounced CNS side effects as well as anticholinergic effects including dry mouth. The newly developed medications are mostly $\mathrm{H} 1$ receptor antagonists, and although they are not completely free from side effects, they did show a significantly lower number of cases involving dry mouth. Second-generation antihistamines include cetirizine, acrivastine, astemizole, loratadine and mizolastine [15].

\section{H2 receptor antagonists and proton-pump inhibitors}

The most common method for the eradication of Helicobacter pylori is treatment with three main agents: amoxicillin or tetracycline, metronidazole, and a bismuth derivative. A controlled trial has investigated whether the addition of $\mathrm{H} 2$ receptor antagonist such as ranitidine, can accelerate the treatment process, and results demonstrated that this was indeed effective for the eradication of the bacteria. Unfortunately, addition of ranitidine increased incidence of several side effects including dry mouth, found in $41 \%$ of recipients [37]. Proton-pump inhibitors are used to decrease the volume of gastric juice and acid secretion. Drugs in this group, such as omeprazole, act by irreversibly inhibiting the hydrogen/potassium ATPase enzymes, found on gastric parietal cells. A study evaluating the usage of omeprazole reported that several patients administered omeprazole for more than six weeks complained of a dry mouth [38]. In addition, four out of the six patients experiencing xerostomia had altered salivary flow rates while undergoing treatment, and after cessation of the treatment, flow rates returned to normal. When 
saliva from the patients was cultured, significant amounts of Candida albicans were able to grow in the cultured saliva. These three patients out of the six were the ones with the lowest flow rates. Growth of Staphylococcus aureus was also recorded in one of the samples. The conclusion drawn from this study was that despite the correction of salivary flow rates upon cessation of omeprazole use, a dangerous window inviting opportunistic infections is open during the period of usage, especially in edentulous patients [38].

\section{Opioids and benzodiazepines}

Opioids such as morphine and benzodiazepines are commonly used to facilitate patient comfort and aid in mechanical ventilation. Unfortunately, none of these agents embody all the characteristics of an ideal sedative or analgesic agent, so they are given in combination [39]. Opioids are well-known to cause dry mouth. For example, a study investigating morphine responses in human patients reported a high incidence of dry mouth [40]. Similarly, a study evaluating controlled-release morphine sulfate reported several side effects including dry mouth [41]. Another opioid, dihydrocodeine, has been reported to cause dry mouth in $80 \%$ of the patients receiving it [42]. Tramadol, a synthetic, centrally acting analgesic agent, has also displayed dry mouth as one of its most common adverse reactions, with an incidence of $1.6-6.1 \%$ [43]. Benzodiazepines are widely used as anxiolytics, hypnotics, anticonvulsants and muscle relaxants. They act by enhancing GABA-A receptor function in the CNS [44]. Members of this group, such as diazepam, may also cause a slight dry mouth sensation [45].

\section{Cytotoxic agents}

Administration of cytotoxic drugs may result in complicated oral manifestations. The major effect of cytotoxic agents is suppression of the immune system, but they can also directly damage the oral mucosa. For example, the common cytotoxic drugs 5-flurourcail, is frequently associated with xerostomia [4]. Xerostomia during chemotherapy is also a major reason for the development of mucositis likely due to the low level of neutrophils caused by immunosuppressing medications [46]. This damage caused by cytotoxic drugs can be intensified when considering xerostomia and secondary infections [47]. A study comparing healthy patients with patients undergoing chemotherapy found differences in the characteristics of saliva between the two groups [47]. Based on these findings, a more elaborate study was initiated in patients taking cytotoxic medications to evaluate the volume and composition of saliva as well as potential pathogens in the oral cavity. Twelve patients were evaluated before and after the beginning of cytotoxic therapy. They were also evaluated four weeks and twelve weeks after the cessation of treatment. In samples collected twelve weeks after treatment was completed, the amounts of saliva was significantly reduced. The composition of the saliva was also altered, and important components such as Immunoglobulin A and amylase were decreased. The only component of saliva that remained within normal value was lysozyme. The study also reported large growth of Candida species in the saliva as well as $S$. aureus in some samples. This study concluded that cytotoxic chemotherapy is a leading cause for reduced salivary flow, altered saliva composition, and an oral environment that allowed for increased growth of opportunistic pathogens [47]. 


\section{DISCUSSION AND CONCLUSIONS}

Although an immense number of therapeutic agents can cause undesirable oral side effects and many of these agents are used to treat very common conditions, the importance of oral side effects of medications is underestimated by dentists. In addition, drug-induced oral adverse reactions are a common issue raised by dental patients. In most cases, resolution of these side effects can be achieved by withdrawal and replacement of the agent in question, coupled with a symptomatic therapy. Unfortunately cessation or replacement of the medication is not always an option. As such, best dental practice should include an extensive knowledge of possible complications, careful patient monitoring, and proper case management. Treatment of symptoms substitution or withdrawal of responsible medications are important, but they are not the only steps in a treatment plan. Maintenance of good oral hygiene, cessation of bad habits such as alcohol consumption and smoking, adequate nutrition and vitamin intake, and proper hydration can all influence the extent of the undesirable effects of medication usage [48]. As one of the most common oral reactions related to medication usage, xerostomia also has several pharmaceutical treatments such as saliva stimulants. After carefully considering the mechanisms and likely causes of xerostomia, the dentist should develop an individual and appropriate treatment plan for each affected patient. Some saliva stimulants require a prescription, while some do not. These agents come in the form of mouthwash, saliva substitute gels, toothpastes, patches and even sugar-free chewing gums, and can assist in the relief of dry mouth sensation. However, the use of such therapeutic agents does not always result in relief of symptoms, and it does not enable a higher-dose of the reaction-inducing medication [49]. Lastly, it is important to highlight that the field of pharmacology progresses rapidly as new therapies are developed and clinicians should pay special attention to the therapeutic agents used by their patients. It is necessary to understand both the mechanisms by which the drugs act, as well as their potential adverse effects [50].

\section{ACKNOWLEDGMENT}

This paper was published as part of the "Translational Geroscience" initiative of Physiology International [51-61].

\section{REFERENCES}

1. Bhattarai KR, Junjappa R, Handigund M, Kim HR, Chae HJ. The imprint of salivary secretion in autoimmune disorders and related pathological conditions. Autoimmun Rev 2018; 17: 376-90.

2. Proctor GB. The physiology of salivary secretion. Periodontology 2000 2016; 70: 11-25.

3. Ekström J, Khosravani N, Castagnola M, Messana I. Saliva and the control of its secretion. In: Ekberg O, editor. Dysphagia: diagnosis and treatment. Berlin, Heidelberg: Springer Berlin Heidelberg; 2012. p. 19-47.

4. Scully C. Drug effects on salivary glands: dry mouth. Oral Dis 2003; 9: 165-76.

5. Kawaguchi M, Yamagishi H. [Receptive systems for drugs in salivary gland cells]. Nihon Yakurigaku Zasshi 1995; 105: 295-303. 
6. Abdollahi M, Radfar M. A review of drug-induced oral reactions. J Contemp Dent Pract 2003; 4: 10-31.

7. Szabadi E, Tavernor S. Hypo- and hypersalivation induced by psychoactive drugs. CNS Drugs 1999; 11 : 449-66.

8. An JY, Darveau R, Kaeberlein M. Oral health in geroscience: animal models and the aging oral cavity. Geroscience 2018; 40: 1-10.

9. Villa A, Connell CL, Abati S. Diagnosis and management of xerostomia and hyposalivation. Ther Clin Risk Manag 2015; 11: 45-51.

10. Singh H, Torralba MG, Moncera KJ, DiLello L, Petrini J, Nelson KE, et al. Gastro-intestinal and oral microbiome signatures associated with healthy aging. Geroscience 2019; 41: 907-21.

11. Hunter KD, Wilson WS. The effects of antidepressant drugs on salivary flow and content of sodium and potassium ions in human parotid saliva. Arch Oral Biol 1995; 40: 983-9.

12. Pomara N, Shao B, Choi SJ, Tun H, Suckow RF. Sex-related differences in nortriptyline-induced side-effects among depressed patients. Prog Neuropsychopharmacol Biol Psychiatry 2001; 25: 1035-48.

13. Mangrella M, Motola G, Russo F, Mazzeo F, Giassa T, Falcone G, et al. [Hospital intensive monitoring of adverse reactions of ACE inhibitors]. Minerva Med 1998; 89: 91-7.

14. Thorn CF, Ellison DH, Turner ST, Altman RB, Klein TE. PharmGKB summary: diuretics pathway, pharmacodynamics. Pharmacogenet Genomics 2013; 23: 449-53.

15. Scully C, Bagan JV. Adverse drug reactions in the orofacial region. Crit Rev Oral Biol Med 2004; 15: 221-39.

16. Atkinson JC, Shiroky JB, Macynski A, Fox PC. Effects of furosemide on the oral cavity. Gerodontology 1989; 8: $23-6$.

17. Hattori T, Wang PL. Calcium antagonists cause dry mouth by inhibiting resting saliva secretion. Life Sci 2007; 81: 683-90.

18. Blair DT, Dauner A. Extrapyramidal symptoms are serious side-effects of antipsychotic and other drugs. Nurse Pract 1992; 17: 56, 62-4, 67.

19. Keks NA. Minimizing the non-extrapyramidal side-effects of antipsychotics. Acta Psychiatr Scand Suppl 1996; 389: 18-24.

20. Duggan L, Fenton M, Rathbone J, Dardennes R, El-Dosoky A, Indran S. Olanzapine for schizophrenia. Cochrane Database Syst Rev 2005: Cd001359.

21. Tollefson GD, Birkett MA, Kiesler GM, Wood AJ. Double-blind comparison of olanzapine versus clozapine in schizophrenic patients clinically eligible for treatment with clozapine. Biol Psychiatry 2001; 49: 52-63.

22. Fulton B, Goa KL. Olanzapine. A review of its pharmacological properties and therapeutic efficacy in the management of schizophrenia and related psychoses. Drugs 1997; 53: 281-98.

23. Mullen J, Jibson MD, Sweitzer D. A comparison of the relative safety, efficacy, and tolerability of quetiapine and risperidone in outpatients with schizophrenia and other psychotic disorders: the quetiapine experience with safety and tolerability (QUEST) study. Clin Ther 2001; 23: 1839-54.

24. Jacobsen FM, Comas-Diaz L. Donepezil for psychotropic-induced memory loss. J Clin Psychiatry 1999; 60: 698-704.

25. Shashikiran ND, Reddy VV, Raju PK. Effect of antiasthmatic medication on dental disease: dental caries and periodontal disease. J Indian Soc Pedod Prev Dent 2007; 25: 65-8.

26. Koumis T, Samuel S. Tiotropium bromide: a new long-acting bronchodilator for the treatment of chronic obstructive pulmonary disease. Clin Ther 2005; 27: 377-92.

27. Casaburi R, Briggs , Jr DD, Donohue JF, Serby CW, Menjoge SS, Witek, Jr TJ. The spirometric efficacy of once-daily dosing with tiotropium in stable COPD: a 13-week multicenter trial. The US Tiotropium Study Group. Chest 2000; 118: 1294-302. 
28. Tyagi N, Gulati K, Vijayan V, Ray A. A study to monitor adverse drug reactions in patients of chronic obstructive pulmonary disease: focus on theophylline. Indian J Chest Dis Allied Sci 2008; 50: 199-202.

29. Thomson WM, Spencer AJ, Slade GD, Chalmers JM. Is medication a risk factor for dental caries among older people? Community Dent Oral Epidemiol 2002; 30: 224-32.

30. Nabi G, Cody JD, Ellis G, Herbison P, Hay-Smith J. Anticholinergic drugs versus placebo for overactive bladder syndrome in adults. Cochrane Database Syst Rev 2006: Cd003781.

31. Garely AD, Burrows LJ. Current pharmacotherapeutic strategies for overactive bladder. Expert Opin Pharmacother 2002; 3: 827-33.

32. Yarker YE, Goa KL, Fitton A. Oxybutynin. Drugs Aging 1995; 6: 243-62.

33. Wagstaff AJ, Bryson HM. Tizanidine. A review of its pharmacology, clinical efficacy and tolerability in the management of spasticity associated with cerebral and spinal disorders. Drugs 1997; 53: 435-52.

34. Stien R, Nordal HJ, Oftedal SI, Slettebo M. The treatment of spasticity in multiple sclerosis: a double-blind clinical trial of a new anti-spastic drug tizanidine compared with baclofen. Acta Neurol Scand 1987; 75: 190-4.

35. Gonzalez MA, Estes KS. Pharmacokinetic overview of oral second-generation H1 antihistamines. Int J Clin Pharmacol Ther 1998; 36: 292-300.

36. Church DS, Church MK. Pharmacology of antihistamines. World Allergy Organ J 2011; 4: S22-7.

37. Kaviani MJ, Malekzadeh R, Vahedi H, Sotoudeh M, Kamalian N, Amini M, et al. Various durations of a standard regimen (amoxycillin, metronidazole, colloidal bismuth sub-citrate for 2 weeks or with additional ranitidine for 1 or 2 weeks) on eradication of Helicobacter pylori in Iranian peptic ulcer patients. A randomized controlled trial. Eur J Gastroenterol Hepatol 2001; 13: 915-9.

38. Teare JP, Spedding C, Whitehead MW, Greenfield SM, Challacombe SJ, Thompson RP. Omeprazole and dry mouth. Scand J Gastroenterol 1995; 30: 216-8.

39. Devlin JW, Roberts RJ. Pharmacology of commonly used analgesics and sedatives in the ICU: benzodiazepines, propofol, and opioids. Crit Care Clin 2009; 25: 431-49, vii.

40. Zacny JP. Morphine responses in humans: a retrospective analysis of sex differences. Drug Alcohol Depend 2001; 63: 23-8.

41. Bruera E, Belzile M, Neumann CM, Ford I, Harsanyi Z, Darke A. Twice-daily versus once-daily morphine sulphate controlled-release suppositories for the treatment of cancer pain. A randomized controlled trial. Support Care Cancer 1999; 7: 280-3.

42. Freye E, Baranowski J, Latasch L. Dose-related effects of controlled release dihydrocodeine on oro-cecal transit and pupillary light reflex. A study in human volunteers. Arzneimittelforschung 2001; 51: 60-6.

43. Scott LJ, Perry CM. Tramadol: a review of its use in perioperative pain. Drugs 2000; 60: 139-76.

44. Mohler H, Fritschy JM, Rudolph U. A new benzodiazepine pharmacology. J Pharmacol Exp Ther 2002; 300: 2-8.

45. Conti L, Pinder RM. A controlled comparative trial of mianserin and diazepam in the treatment of anxiety states in psychiatric out-patients. J Int Med Res 1979; 7: 285-9.

46. McCarthy GM, Awde JD, Ghandi H, Vincent M, Kocha WI. Risk factors associated with mucositis in cancer patients receiving 5-fluorouracil. Oral Oncol 1998; 34: 484-90.

47. Main BE, Calman KC, Ferguson MM, Kaye SB, MacFarlane TW, Mairs RJ, et al. The effect of cytotoxic therapy on saliva and oral flora. Oral Surg Oral Med Oral Pathol 1984; 58: 545-8.

48. Cicek Y, Ertas U. The normal and pathological pigmentation of oral mucous membrane: a review. J Contemp Dent Pract 2003; 4: 76-86.

49. Tincello DG, Adams EJ, Sutherst JR, Richmond DH. Oxybutynin for detrusor instability with adjuvant salivary stimulant pastilles to improve compliance: results of a multicentre, randomized controlled trial. BJU Int 2000; 85: 416-20. 
50. Abdollahi M, Rahimi R, Radfar M. Current opinion on drug-induced oral reactions: a comprehensive review. J Contemp Dent Pract 2008; 9: 1-15.

51. Bagis Z, Ozeren M, Buyukakilli B, Balli E, Yaman S, Yetkin D, et al. Effect of iloprost on contractile impairment and mitochondrial degeneration in ischemia-reperfusion of skeletal muscle. Physiol Int 2018; 105: 61-75.

52. Bin-Jaliah I, Sakr HF. Melatonin ameliorates brain oxidative stress and upregulates senescence marker protein-30 and osteopontin in a rat model of vascular dementia. Physiol Int 2018; 105: 38-52.

53. Bruno JA, Feldman CH, Konas DW, Kerrihard AL, Matthews EL. Incorporating sprouted chickpea flour in pasta increases brachial artery flow-mediated dilation. Physiol Int 2019; 106: 207-12.

54. Hackney AC, Hooper DR. Low testosterone: androgen deficiency, endurance exercise training, and competitive performance. Physiol Int 2019; 106: 379-89.

55. McCormick JJ, VanDusseldorp TA, Ulrich CG, Lanphere RL, Dokladny K, Mosely PL, et al. The effect of aging on the autophagic and heat shock response in human peripheral blood mononuclear cells. Physiol Int 2018; 105: 247-56.

56. Nagy J, Kovacs T. A brief review on the rising incidence of chronic kidney diseases and non-alcoholic fatty liver disease. Physiol Int 2019; 106: 305-10.

57. Nemcsik J, Tabak A, Batta D, Cseprekal O, Egresits J, Tisler A. Integrated central blood pressure-aortic stiffness risk score for cardiovascular risk stratification in chronic kidney disease. Physiol Int 2018; 105: 335-46.

58. Sachse C, Trozic I, Brix B, Roessler A, Goswami N. Sex differences in cardiovascular responses to orthostatic challenge in healthy older persons: a pilot study. Physiol Int 2019; 106: 236-49.

59. Sumida K, Kovesdy CP. The gut-kidney-heart axis in chronic kidney disease. Physiol Int 2019; 106: 195-206.

60. Yabluchanskiy A, Ungvari Z, Csiszar A, Tarantini S. Advances and challenges in geroscience research: an update. Physiol Int 2018; 105: 298-308.

61. Zlibut A, Bocsan IC, Pop RM, Vesa SC, Bheecarry K, Revnic R, et al. Role of pentraxin-3 in risk assessment of patients with metabolic syndrome. Physiol Int 2019; 106: 283-93

Open Access statement. This is an open-access article distributed under the terms of the Creative Commons Attribution 4.0 International License (https://creativecommons.org/licenses/by/4.0/), which permits unrestricted use, distribution, and reproduction in any medium, provided the original author and source are credited, a link to the CC License is provided, and changes if any - are indicated. (SID_1) 